\title{
Nonexpansive mappings on Abelian Banach algebras and their fixed points
}

W Fupinwong*

\author{
"Correspondence: \\ fupinw@chiangmai.ac.th \\ Department of Mathematics, \\ Faculty of Science, Chiang Mai \\ University, Chiang Mai, 50200, \\ Thailand \\ Centre of Excellence in \\ Mathematics, CHE, Si Ayutteaya Rd. \\ Bangkok, 10400, Thailand
}

\begin{abstract}
A Banach space $X$ is said to have the fixed point property if for each nonexpansive mapping $T: E \rightarrow E$ on a bounded closed convex subset $E$ of $X$ has a fixed point. We show that each infinite dimensional Abelian complex Banach algebra $X$ satisfying: (i) property (A) defined in (Fupinwong and Dhompongsa in Fixed Point Theory Appl. 2010:Article ID 34959, 2010), (ii) $\|x\| \leq\|y\|$ for each $x, y \in X$ such that $|\tau(x)| \leq|\tau(y)|$ for each $\tau \in \Omega(X)$, (iii) $\inf \{r(x): x \in X,\|x\|=1\}>0$ does not have the fixed point property. This result is a generalization of Theorem 4.3 in (Fupinwong and Dhompongsa in Fixed Point Theory Appl. 2010:Article ID 34959, 2010).
\end{abstract}

MSC: 46B20; 46J99

Keywords: fixed point property; nonexpansive mapping; Abelian Banach algebra

\section{Introduction}

A Banach space $X$ is said to have the fixed point property (or weak fixed point property) if for each nonexpansive mapping $T: E \rightarrow E$ on a bounded closed convex (or weakly compact convex, resp.) subset $E$ of $X$ has a fixed point.

For the weak fixed point property of certain Banach algebras, Lau et al. [1] showed that the space $C_{0}(G)$, where $G$ is a locally compact group, has the weak fixed point property if and only if $G$ is discrete, and a von Neumann algebra has the weak fixed point property if and only if it is finite dimensional. Benavides and Pineda [2] proved that each $\omega$-almost weakly orthogonal closed subspace of $C\left(K_{1}\right)$, where $K_{1}$ is a metrizable compact space, has the weak fixed point property and $C\left(K_{2}\right)$, where $K_{2}$ is a compact set with $K_{2}^{(\omega)}=\emptyset$, has the weak fixed point property.

As for the fixed point property, Dhompongsa et al. [3] showed that a $C^{\star}$-algebra has the fixed point property if and only if it is finite dimensional. Fupinwong and Dhompongsa [4] proved that each infinite dimensional unital Abelian Banach algebra $X$ with $\Omega(X) \neq \emptyset$ satisfying: (i) (A) defined in [4], (ii) $\|x\| \leq\|y\|$ for each $x, y \in X$ with $|\tau(x)| \leq|\tau(y)|$ for each $\tau \in \Omega(X)$, (iii) $\inf \{r(x): x \in X,\|x\|=1\}>0$ does not have the fixed point property. Alimohammadi and Moradi [5] used the above result to obtain sufficient conditions to show that some unital uniformly closed subalgebras of $C(X)$, where $X$ is a compact space, do not have the fixed point property.

In this paper, we show that the unitality in the result proved in [4] can be omitted.

\section{Preliminaries and lemmas}

We assume that the field of each vector space in this paper is complex.

\section{Springer}

(c) 2012 Fupinwong; licensee Springer. This is an Open Access article distributed under the terms of the Creative Commons Attribution License (http://creativecommons.org/licenses/by/2.0), which permits unrestricted use, distribution, and reproduction in any medium, provided the original work is properly cited. 
Let $X$ be a Banach algebra. Define $\widetilde{X}=X \oplus \mathbb{C}$ and a multiplication on $\widetilde{X}$ by

$$
(a, \lambda)(b, \mu)=(a b+\lambda b+\mu a, \lambda \mu) .
$$

We have $\widetilde{X}$ is a unital Banach algebra with the unit $(0,1)$ and called the unitization of $X . \tilde{X}$ is also Abelian if $X$ is Abelian.

If $\widetilde{X}$ is the unitization of a Banach algebra $X$ and $\Omega(X)$ is the set of characters on $X$, then the set $\Omega(\widetilde{X})$ of characters on $\widetilde{X}$ is equal to

$$
\{\tilde{\tau}: \tau \in \Omega(X)\} \cup\left\{\tau_{\infty}\right\}
$$

where $\tilde{\tau}$ is defined from $\tau \in \Omega(X)$ by

$$
\widetilde{\tau}((a, \lambda))=\tau(a)+\lambda,
$$

for each $(a, \lambda) \in \widetilde{X}$, and $\tau_{\infty}$ is the canonical homomorphism defined by

$$
\tau_{\infty}((a, \lambda))=\lambda
$$

for each $(a, \lambda) \in \tilde{X}$.

If $X$ is an Abelian Banach algebra, condition (A) is defined by:

(A) For each $x \in X$, there exists an element $y \in X$ such that $\tau(y)=\overline{\tau(x)}$, for each $\tau \in \Omega(X)$.

It can be seen that if $X$ satisfies (A), then so does the unitization $\widetilde{X}$ of $X$.

Let $X$ be an Abelian Banach algebra. The Gelfand representation $\varphi: X \rightarrow C(\Omega(X))$ is defined by $x \mapsto \widehat{x}$, where $\widehat{x}$ is defined by

$$
\widehat{x}(\tau)=\tau(x)
$$

for each $\tau \in C(\Omega(X))$.

The following lemma was proved in [4].

Lemma 2.1 Let $X$ be a unital Abelian Banach algebra satisfying $(A)$ and

$$
\inf \{r(x): x \in X,\|x\|=1\}>0 .
$$

Then:

(i) the Gelfand representation $\varphi$ is a bounded isomorphism,

(ii) the inverse $\varphi^{-1}$ is also a bounded isomorphism.

Let $X$ be an Abelian Banach algebra satisfying (A) and $\inf \{r(x): x \in X,\|x\|=1\}>0$. It can be seen that $X$ is embedded in $C(\Omega(\widetilde{X}))$ as the closed subalgebra $Y=\{\widehat{x} \in C(\Omega(\widetilde{X}))$ : $\left.\widehat{x}\left(\tau_{\infty}\right)=0\right\}$. Moreover, for each $x \in \tilde{X}, x$ is in $X$ if and only if $\tau_{\infty}(x)=0$.

Lemma 2.2 Let $X$ be an infinite dimensional Abelian Banach algebra satisfying (A) and

$$
\inf \{r(x): x \in X,\|x\|=1\}>0 .
$$


Then we have:

(i) $\Omega(X)$ is an infinite set.

(ii) If there exists a bounded sequence $\left\{x_{n}\right\}$ in $X$ which contains no convergent subsequences and such that $\left\{\tau\left(x_{n}\right): \tau \in \Omega(X)\right\}$ is finite for each $n \in \mathbb{N}$, then there is an element $x_{0} \in X$ such that $\left\{\omega\left(x_{0}\right): \omega \in \Omega(\widetilde{X})\right\}$ is equal to $\left\{0,1, \frac{1}{2}, \frac{2}{3}, \frac{3}{4}, \ldots\right\}$ or $\left\{0,1, \frac{1}{2}, \frac{1}{3}, \frac{1}{4}, \ldots\right\}$.

(iii) There is an element $x_{0} \in X$ such that $\left\{\omega\left(x_{0}\right): \omega \in \Omega(\widetilde{X})\right\}$ is an infinite set.

(iv) There exists a sequence $\left\{x_{n}\right\}$ in $X$ such that $\left\{\omega\left(x_{n}\right): \omega \in \Omega(\widetilde{X})\right\} \subset[0,1]$, for each $n \in \mathbb{N}$, and $\left\{\left(\widehat{x_{n}}\right)^{-1}\{1\}\right\}$ is a sequence of nonempty pairwise disjoint subsets of $\Omega(\widetilde{X})$.

Proof (i) From Lemma 2.10(i) in [4], we have $\Omega(\widetilde{X})$ is infinite. Since

$$
\Omega(\widetilde{X})=\{\tilde{\tau}: \tau \in \Omega(X)\} \cup\left\{\tau_{\infty}\right\}
$$

where $\tilde{\tau}$ is defined from $\tau \in \Omega(X)$ by $\widetilde{\tau}((a, \lambda))=\tau(a)+\lambda$, for each $(a, \lambda) \in \widetilde{X}$, and $\tau_{\infty}$ is the canonical homomorphism, so $\Omega(X)$ is also infinite.

(ii) Let $\left\{x_{n}\right\}$ be a bounded sequence in $X$ which has no convergent subsequences and the set $\left\{\tau\left(x_{n}\right): \tau \in \Omega(X)\right\}$ be finite for each $n \in \mathbb{N}$. Consider $\left\{x_{n}\right\}$ a sequence in $\widetilde{X}$, so $\left\{\omega\left(x_{n}\right)\right.$ : $\omega \in \Omega(\widetilde{X})\}$ is finite for each $n \in \mathbb{N}$. It follows from the proof of Lemma 2.10(ii) in [4] that

$$
\Omega(\widetilde{X})=\left(\bigcup_{n \in \mathbb{N}} G_{n}\right) \cup F
$$

where $F$ is a closed set in $\Omega(\widetilde{X}), G_{n}$ is closed and open for each $n \in \mathbb{N}$, and $\left\{F, G_{1}, G_{2}, \ldots\right\}$ is a partition of $\Omega(\widetilde{X})$. There are two cases to be considered. If $\tau_{\infty}$ is in $F$, defined $\psi: \Omega(\widetilde{X}) \rightarrow \mathbb{R}$ by

$$
\psi(\tau)= \begin{cases}1, & \text { if } \tau \in G_{1}, \\ \frac{1}{n}, & \text { if } \tau \in G_{n}, n \geq 2, \\ 0, & \text { if } \tau \in F .\end{cases}
$$

If $\tau_{\infty}$ is in $G_{n_{0}}$, for some $n_{0} \in \mathbb{N}$, we may assume that $n_{0}=1$, defined $\psi: \Omega(\widetilde{X}) \rightarrow \mathbb{R}$ by

$$
\psi(\tau)= \begin{cases}0, & \text { if } \tau \in G_{1}, \\ \frac{n-1}{n}, & \text { if } \tau \in G_{n}, n \geq 2, \\ 1, & \text { if } \tau \in F .\end{cases}
$$

For each case, we have the inverse image of each closed set in $\psi(\Omega(\widetilde{X}))$ is closed, so $\psi \in C(\Omega(\widetilde{X}))$. Let $\varphi: \widetilde{X} \rightarrow C(\Omega(\widetilde{X}))$ be the Gelfand representation. Therefore, $\varphi^{-1}(\psi)$ is an element in $\widetilde{X}$, say $x_{0}$, such that $\left\{\omega\left(x_{0}\right): \omega \in \Omega(\widetilde{X})\right\}$ is equal to $\left\{0,1, \frac{1}{2}, \frac{2}{3}, \frac{3}{4}, \ldots\right\}$ or $\left\{0,1, \frac{1}{2}, \frac{1}{3}, \frac{1}{4}, \ldots\right\}$. We have $x_{0} \in X$ since $\tau_{\infty}\left(x_{0}\right)=\psi\left(\tau_{\infty}\right)=0$.

(iii) Assume to the contrary that $\{\omega(x): \omega \in \Omega(\widetilde{X})\}$ is finite for each $x \in X$. Since $X$ is infinite dimensional, so there is a bounded sequence $\left\{x_{n}\right\}$ in $X$ which has no convergent subsequences. Thus $\left\{\omega\left(x_{n}\right): \omega \in \Omega(\widetilde{X})\right\}$ is finite for each $n \in \mathbb{N}$. It follows from (ii) that there exists $x_{0} \in X$ such that $\left\{\omega\left(x_{0}\right): \omega \in \Omega(\widetilde{X})\right\}$ is infinite. This leads to a contradiction. 
(iv) It follows from (iii) that there exists an element $x_{1} \in X$ such that $\left\{\omega\left(x_{1}\right): \omega \in \Omega(\widetilde{X})\right\}$ is infinite. We may assume that there exists a strictly decreasing sequence of real numbers $\left\{a_{n}\right\}$ such that

$$
\left\{a_{n}\right\} \subset \widehat{x_{1}}(\Omega(\widetilde{X})) \subset[0,1], \quad a_{1}<1,
$$

and $\omega\left(x_{1}\right)=1$ for some $\omega \in \Omega(\widetilde{X})$. Define $g_{1}:[0,1] \rightarrow[0,1]$ by

$$
g_{1}(t)= \begin{cases}\frac{t}{a_{1}}, & \text { if } t \in\left[0, a_{1}\right], \\ 1+\frac{\left(g_{1}\left(a_{2}\right)-1\right)\left(t-a_{1}\right)}{2\left(1-a_{1}\right)}, & \text { if } t \in\left[a_{1}, 1\right] .\end{cases}
$$

So $g_{1}$ is a continuous function joining the points $(0,0)$ and $\left(a_{1}, 1\right)$, and $g_{1}(1) \in\left(g_{1}\left(a_{2}\right), 1\right)$. Let $\widehat{x_{2}}=g_{1} \circ \widehat{x_{1}}$, and define a continuous function $g_{2}:[0,1] \rightarrow[0,1]$ by

$$
g_{2}(t)= \begin{cases}\frac{t}{g_{1}\left(a_{2}\right)}, & \text { if } t \in\left[0, g_{1}\left(a_{2}\right)\right], \\ 1+\frac{\left(g_{2}\left(g_{1}\left(a_{3}\right)\right)-1\right)\left(t-g_{1}\left(a_{2}\right)\right)}{2\left(1-g_{1}\left(a_{2}\right)\right)}, & \text { if } t \in\left[g_{1}\left(a_{2}\right), 1\right] .\end{cases}
$$

$g_{2}$ is joining the point $(0,0)$ and $\left(g_{1}\left(a_{2}\right), 1\right)$ and $g_{2}(1) \in\left(g_{2}\left(g_{1}\left(a_{3}\right)\right), 1\right)$. Let $\widehat{x_{3}}=g_{2} \circ \widehat{x_{2}}$. Continuing in this process, we obtain a sequence of points $\left\{x_{n}\right\}$ in $\widetilde{X}$ with $\left\{\omega\left(x_{n}\right): \omega \in \Omega(X)\right\} \subset$ $[0,1]$, for each $n \in \mathbb{N}$, and $\left\{\left(\widehat{x_{n}}\right)^{-1}\{1\}\right\}$ is a sequence of nonempty pairwise disjoint subsets of $\Omega(\widetilde{X})$. Since $g_{n}(0)=0$, for each $n \in \mathbb{N}$, so

$$
\widehat{x_{i+1}}\left(\tau_{\infty}\right)=\left(g_{i} \circ \cdots \circ g_{1} \circ \widehat{x_{1}}\right)\left(\tau_{\infty}\right)=\left(g_{i} \circ \cdots \circ g_{1}\right)(0)=0
$$

for each $i \in \mathbb{N}$. Then $\tau_{\infty}\left(x_{n}\right)=0$, for each $n \in \mathbb{N}$. Thus $\left\{x_{n}\right\}$ is a sequence in $X$.

\section{Main theorem}

Theorem 3.1 Let $X$ be an infinite dimensional Abelian Banach algebra satisfying $(A)$ and each of the following:

(i) If $x, y \in X$ is such that $|\tau(x)| \leq|\tau(y)|$, for each $\tau \in \Omega(X)$, then $\|x\| \leq\|y\|$,

(ii) $\inf \{r(x): x \in X,\|x\|=1\}>0$.

Then $X$ does not have the fixed point property.

Proof Assume to the contrary that $X$ has the fixed point property. From Lemma 2.2(iv), there exists a sequence $\left\{x_{n}\right\}$ in $X$ such that $\left\{\omega\left(x_{n}\right): \omega \in \Omega(\widetilde{X})\right\} \subset[0,1]$ for each $n \in \mathbb{N}$, and $\left\{\left(\widehat{x_{n}}\right)^{-1}\{1\}\right\}$ is a sequence of nonempty pairwise disjoint subsets of $\Omega(\widetilde{X})$. Let $A_{n}=\left(\widehat{x_{n}}\right)^{-1}\{1\}$, and define $T_{n}: E_{n} \rightarrow E_{n}$ by

$$
x \mapsto x_{n} x,
$$

where

$$
E_{n}=\left\{x \in X: 0 \leq \omega(x) \leq 1 \text { for each } \omega \in \Omega(\widetilde{X}) \text {, and } \omega(x)=1 \text { if } \omega \in A_{n}\right\} .
$$

From (i) and (ii), $T_{n}: E_{n} \rightarrow E_{n}$ is a nonexpansive mapping on the bounded closed convex set $E_{n}$ for each $n \in \mathbb{N}$. Indeed, $E_{n}$ is bounded since

$$
\inf \{r(x): x \in X,\|x\|=1\} \leq r\left(\frac{x}{\|x\|}\right)=\sup _{\omega \in \Omega(\widetilde{X})}\left|\omega\left(\frac{x}{\|x\|}\right)\right|=\frac{1}{\|x\|} \sup _{\omega \in \Omega(\widetilde{X})}|\omega(x)|
$$


for each $x \in X$. So $T_{n}$ has a fixed point, say $y_{n}$, for each $n \in \mathbb{N}$. We have $y_{n}=x_{n} y_{n}$, hence $\widehat{y_{n}}=\widehat{x_{n}} \widehat{y_{n}}$, and then

$$
\widehat{y_{n}}(\omega)= \begin{cases}0, & \text { if } \omega \text { is not in } A_{n}, \\ 1, & \text { if } \omega \text { is in } A_{n},\end{cases}
$$

for each $n \in \mathbb{N}$. We have $\left\|\widehat{y_{m}}-\widehat{y_{n}}\right\|=1$, if $m \neq n$, since $A_{1}, A_{2}, A_{3}, \ldots$ are pairwise disjoint. Therefore, $\left\{\widehat{y_{n}}\right\}$ has no convergent subsequences. From Lemma 2.1, $\widetilde{X}$ and $C(\Omega(\widetilde{X}))$ are homeomorphic. So $\left\{y_{n}\right\}$ has no convergent subsequences. From Lemma 2.2(ii), there exists an element $x_{0}$ in $X$ such that $\left\{\omega\left(x_{0}\right): \omega \in \Omega(\widetilde{X})\right\}$ is equal to $\left\{0,1, \frac{1}{2}, \frac{2}{3}, \frac{3}{4}, \ldots\right\}$ or $\left\{0,1, \frac{1}{2}, \frac{1}{3}, \frac{1}{4}, \ldots\right\}$. Let $A_{0}=\left(\widehat{x_{0}}\right)^{-1}\{1\}$. Define $T_{0}: E_{0} \rightarrow E_{0}$ by

$$
x \mapsto x_{0} x,
$$

where

$$
E_{0}=\left\{x \in X: 0 \leq \omega(x) \leq 1 \text { for each } \omega \in \Omega(\widetilde{X}) \text {, and } \omega(x)=1 \text { if } \omega \in A_{0}\right\} .
$$

From (i) and (ii), $T_{0}$ is a nonexpansive mapping on the bounded closed convex set $E_{0}$. Hence $T_{0}$ has a fixed point, say $y_{0}$, i.e., $y_{0}=x_{0} y_{0}$. Therefore, $\widehat{y_{0}}=\widehat{x_{0}} \widehat{y_{0}}$. Then

$$
\widehat{y_{0}}(\omega)= \begin{cases}0, & \text { if } \omega \text { is not in } A_{0}, \\ 1, & \text { if } \omega \text { is in } A_{0} .\end{cases}
$$

Since $\widehat{y_{0}}=\widehat{x_{0}} \widehat{y_{0}}$, so we have $A_{0}=\left(\widehat{y_{0}}\right)^{-1}\{1\}$ and $\Omega(\widetilde{X}) \backslash A_{0}=\left(\widehat{y_{0}}\right)^{-1}\{0\}$. Then $\Omega(\widetilde{X})$ is a disjoint union of two compact sets $A_{0}$ and $\Omega(\widetilde{X}) \backslash A_{0}$. If

$$
\left\{\omega\left(x_{0}\right): \omega \in \Omega(\widetilde{X})\right\}=\left\{0,1, \frac{1}{2}, \frac{2}{3}, \frac{3}{4}, \ldots\right\}
$$

then $\left\{\left(\widehat{x_{0}}\right)^{-1}\left\{\frac{n}{n+1}\right\}: n \in \mathbb{N}\right\} \cup\left\{\left(\widehat{x_{0}}\right)^{-1}\{0\}\right\}$ is a pairwise disjoint open covering of the compact set $\Omega(\widetilde{X}) \backslash A_{0}$. This leads to a contradiction. Similarly, if

$$
\left\{\omega\left(x_{0}\right): \omega \in \Omega(\widetilde{X})\right\}=\left\{0,1, \frac{1}{2}, \frac{1}{3}, \frac{1}{4}, \ldots\right\},
$$

then $A_{0}$ has a pairwise disjoint open covering, which is a contradiction. So we conclude that $X$ does not have the fixed point property.

The following question is interesting.

Question 3.2 Does the Fourier algebra $A(G)$ or the Fourier-Stieltjes algebra $B(G)$ of a locally compact group $\mathrm{G}$ have property (A) when $G$ is an infinite group?

Note that $A(G)$ or $B(G)$ are both commutative semigroup Banach algebras with the fixed point property if and only if $G$ is finite (see Theorem 5.7 and Corollary 5.8 of [6]). It is well known that $A(G)$ is norm dense in $C_{0}(G)$ with spectrum $G$. 


\section{Competing interests}

The author declares that they have no competing interests.

\section{Acknowledgements}

This research was supported by the Centre of Excellence in Mathematics, the Commission on Higher Education, Thailand.

Received: 25 June 2012 Accepted: 24 August 2012 Published: 14 September 2012

\section{References}

1. Lau, AT, Mah, PF, Ülger, A: Fixed point property and normal structure for Banach spaces associated to locally compact groups. Proc. Am. Math. Soc. 125, 2021-2027 (1997)

2. Domínguez Benavides, T, Japón Pineda, MA: Fixed points of nonexpansive mappings in spaces of continuous functions. Proc. Am. Math. Soc 133, 3037-3046 (2005)

3. Dhompongsa, S, Fupinwong, W, Lawton, W: Fixed point properties of $C^{*}$-algebra. J. Math. Anal. Appl. 374, 22-28 (2011)

4. Fupinwong, W, Dhompongsa, S: The fixed point property of unital abelian Banach algebras. Fixed Point Theory Appl. 2010, Article ID 34959 (2010)

5. Alimohammadi, D, Moradi, S: On the fixed point property of unital uniformly closed subalgebras of $C(X)$. Fixed Point Theory Appl. 2010, Article ID 268450 (2010)

6. Lau, AT, Leinert, M: Fixed point property and the Fourier algebra of a locally compact group. Trans. Am. Math. Soc $360(12), 6389-6402(2008)$

doi:10.1186/1687-1812-2012-150

Cite this article as: Fupinwong: Nonexpansive mappings on Abelian Banach algebras and their fixed points. Fixed Point Theory and Applications 2012 2012:150.

\section{Submit your manuscript to a SpringerOpen ${ }^{\circ}$ journal and benefit from:}

- Convenient online submission

Rigorous peer review

- Immediate publication on acceptance

- Open access: articles freely available online

- High visibility within the field

- Retaining the copyright to your article 